\title{
Output Feedback Stabilization for MIMO Semi-linear Stochastic Systems with Transient Optimisation
}

\author{
Qichun Zhang ${ }^{1} \quad$ Liang $\mathrm{Hu}^{2} \quad \mathrm{John} \mathrm{Gow}^{1}$ \\ ${ }^{1}$ School of Engineering and Sustainable Development, De Montfort University, Leicester, LE1 9BH, UK \\ ${ }^{2}$ School of Computer Science and Informatics, De Montfort University, Leicester, LE1 9BH, UK
}

\begin{abstract}
This paper investigates the stabilisation problem and consider transient optimisation for a class of the multi-inputmulti-output (MIMO) semi-linear stochastic systems. A control algorithm is presented via an $m$-block backstepping controller design where the closed-loop system has been stabilized in a probabilistic sense and the transient performance is optimisable by optimised by searching the design parameters under the given criterion. In particular, the transient randomness and the probabilistic decoupling will be investigated as case studies. Note that the presented control algorithm can be potentially extended as a framework based on the various performance criteria. To evaluate the effectiveness of this proposed control framework, a numerical example is given with simulation results. In summary, the key contributions of this paper are stated as follows: (1) one block backstepping-based output feedback control design is developed to stabilize the dynamic MIMO semi-linear stochastic systems using a linear estimator; (2) the randomness and probabilistic couplings of the system outputs have been minimized based on the optimisation of the design parameters of the controller; (3) a control framework with transient performance enhancement of multi-variable semi-linear stochastic systems has been discussed.
\end{abstract}

Keywords: MIMO Stochastic systems, output feedback stabilisation, block backstepping, randomness attenuation, probabilistic decoupling, mutual information.

\section{Introduction}

Since backstepping was first presented in $1995^{[1]}$, it has been developed as an ideal approach to cover the nonlinearities of dynamic systems. Naturally, the backstepping design can also be adopted for stochastic systems where the system can be represented using the Itô process. In particular, Liu et al. investigated the decentralized control method for SISO stochastic systems ${ }^{[2]}$. Wang et al. developed an adaptive quantized controller via the backstepping and small-gain approach ${ }^{[3]}$. Moreover, the unknown dead-zone and unmodelled dynamics have been considered ${ }^{[4]}$ using adaptive neural network output feedback design. Xie et al. presented a backstepping design for high-order stochastic non-linear systems ${ }^{[5]}$ while the time-varying systems were investigated ${ }^{[6]}$. In addition, incremental stability has been used ${ }^{[7]}$ for backstepping design, and even the stochastic system in non-strict feedback formats have been designed based on backstepping approach ${ }^{[8]}$, etc. Notice that almost all the mentioned methods focused on the single system output.

Motivated by the block backstepping design ${ }^{[9,10]}$ which was introduced by Chang and Cheng et al., the multivariable stochastic systems can be investigated. In particular, Zhang et al. presented a block backstepping design for bilinear stochastic systems ${ }^{[11]}$. However, the transient performance was only optimised in the second moment sense using covariance. As a typical problem of multivariable systems, the couplings between the system outputs will strongly affect the system performance ${ }^{[12]}$, there-

Regular paper

Manuscript received date; revised date

This work was supported by Higher Education Innovation Fund (HEIF 2018-2020), De Montfort University, Leicester, UK.

Recommended by Associate Editor xxxx fore the decoupling design would be a key component for multi-variable system control. For stochastic systems, the probabilistic decoupling ${ }^{[13]}$ was firstly presented in 2015 while the joint probability density function and mutual information ${ }^{[14]}$ were used to deal with the randomness of the investigated systems. Notice that the probabilistic decoupling characterises the full properties of the random variable which means that it contains more information than controlling the covariance only ${ }^{[15]}$. Probabilistic decoupling has been used for neural system modelling where the neural interactions among the axons have been described by the coupling factors from the view of the probabilistic sense $[16,17]$.

On the other hand, the randomness attenuation should also be considered as a part of the transient performance even though the stochastic system can be stabilized with probability one. Various design approaches can achieve differing performance due to the effects of the random noise and the nonlinearities. For a Gaussian distribution, the variance control is widely used ${ }^{[15]}$. Note that the probability density function of the system outputs will not obey the Gaussian distribution even if the system outputs are described by the Itô stochastic differential equation. The system output statistical properties cannot be adequately reflected sufficiently if only the variance and covariance are adopted. To characterise the randomness of the nonGaussian variables, the concept of the entropy has been introduced into the stochastic control system in 2002 by Wang and Yue ${ }^{[18,19]}$. As an optimisation problem, the randomness can be attenuated if the minimum entropy of the system outputs has been achieved. Therefore, the transient performance of the system outputs can be enhanced via minimising the entropy. In practice, entropy optimisation is widely used for network systems ${ }^{[20,21]}$, filter design 
[22, 23] and fault diagnosis $[24,25]$.

Since the semi-linear stochastic systems became a significant research topic affecting many aspects of the applications ${ }^{[26]}$, Zhang et al. presented the output feedback stabilisation algorithm for semi-linear stochastic systems with randomness attenuation ${ }^{[27]}$. Following the discussion above, this paper considers the semi-linear stochastic systems with a transient performance enhancement problem, including output transient randomness attenuation and the output decoupling in a probabilistic sense. Thus, this extension can be considered as a framework where the main challenges are the Itô correction term and probability decoupling. Basically, there is no existing result for probabilistic decoupling design for semi-linear stochastic systems.

In particular, output feedback stabilisation can be achieved by block backstepping with design parameters. In particular, the states of the system can be estimated using the full-state observer design while the new strict format can be delivered considering the estimation error as extended system states. Based on the new system description, the backstepping design can be achieved. Notice that the free parameters in the backstepping design can be further optimised for various performance objectives. In this paper, entropy and mutual information have been used as the performance criteria where the output randomness can be expressed by system outputs' entropy and the minimum of the mutual information can be considered as increasing the independence of the system outputs. Basically, the analytical solutions for probability density functions, entropy and mutual information are very difficult to obtain, the estimation approach can be taken into account using the sampling data. Particularly, we can take the sampling operation for the transient response, then the kernel density estimation $(\mathrm{KDE})^{[28]}$ can be adopted using the Gaussian kernels and the collected data. Following this approach, the probability density function of the system outputs can be approximated by a sliding window. Once the probability density function is obtained, the entropy and mutual information can be calculated simply. To simplify the estimation, the entropy and mutual information can be transformed using information potentials of the system variables. Similarly, the information potentials can also be approximated using the sampling data via kernel density estimation (KDE). All the discussions show that the presented framework is implementable in practice since the data-based performance optimisation is widely used in control applications such as the wind turbine ${ }^{[29,30]}$, internet of things (IoT) ${ }^{[31]}$, Robot-environment interaction ${ }^{[32]}$, teleoperated robots ${ }^{[33]}$, etc.

To illustrate the structure of this paper, its contents have been organised as follows: In Section 2, the preliminaries have been indicated including the investigated system model, control objective, the concept of stability in a probabilistic sense, kernel density estimation, entropy and information potentials, etc. Based on $m$-block backstepping design, the observer-based output feedback controller has been proposed in Section 3 which consists of a linear estimator, block backstepping and probabilistic stability analysis. After that the parametric optimization and controller design procedure are given in Section 4 where the randomness attenuation and probabilistic decoupling are achieved by searching optimal parameters. In order to validate the advantages of the presented control algorithm, simulation results are demonstrated in Section 5. In the end, the paper is summarised and concludes in Section 6 .

\section{Preliminaries}

\subsection{Problem Description}

Consider the following MIMO semi-linear stochastic systems in $m$ blocks strict feedback format, the formulation of the model is shown below:

$$
\begin{aligned}
d \bar{x}_{i} & =\left(A_{i} \bar{x}_{i}+\bar{x}_{i+1}\right) d t+G_{1}\left(\bar{x}_{1}\right) d \beta_{t}, i=1, \cdots, m-1 \\
d \bar{x}_{m} & =\left(A_{m} \bar{x}_{m}+\bar{u}\right) d t+G_{m}\left(\bar{x}_{1}\right) d \beta_{t} \\
\bar{y} & =\bar{x}_{1}
\end{aligned}
$$

where $\beta_{t} \in \mathbb{R}^{s}$ is the Wiener process, $\bar{x}_{i} \in \mathbb{R}^{n}$ is the state for $i$-th block, $A_{i}$ denotes the appropriate dimensional coefficient matrices, $G_{i}: \mathbb{R}^{n} \rightarrow \mathbb{R}^{n}$ are general non-linear functions. $\bar{y}$ and $\bar{u}$ stand for the system output and the control input, respectively. Denoting $\Omega$ as the sample space of continuous functions, $\mathscr{F}$ as a filtration adapted to the Wiener process $\beta_{t}$, and $\mathrm{P}$ as the reference probability measure on $\Omega$, then the triple $(\Omega, \mathscr{F}, \mathrm{P})$ is used to describe the underlying probability space.

Due to the fact that the investigated block-based system is described in strict-feedback form, the system outputs and the control inputs are of the equal dimension. As introduced in Section 1, the control objective is to stabilize the investigated stochastic system in a probabilistic sense while enhancing the transient performance of the stochastic outputs. To analyse the stability of the system, suppose that the following assumption is satisfied for the non-linear function $G_{i}(\cdot)$.

Assumption 1. There exists a positive real constant $\sigma_{i}$, such that the non-linear function $G_{i}(\cdot)$ for $i$-th block of the investigated semi-linear stochastic system(1) meets the following condition:

$$
\left\|G_{i}\left(X_{i}\right)\right\|_{2} \leq \sigma_{i}
$$

where $\|\cdot\|_{2}$ stands for the matrix Euclidean norm.

Remark 1. Notice that we don't impose restriction that the $G_{i}(0)=0$ which means that the outputs of the stochastic system are bounded in a probabilistic sense only using Assumption 1.

\subsection{Stability in a Probabilistic Sense}

Consider the following stochastic non-linear system:

$$
d x=p(x) d t+q(x) d v
$$

where $x \in \mathbb{R}^{n}$ denotes the system state, $v \in \mathbb{R}^{n}$ denotes an independent standard Wiener process, the underlying probability space is the triple $(\Omega, \mathscr{F}, \mathrm{P})$, and $p: \mathbb{R}^{n} \rightarrow \mathbb{R}^{n}$ and $q: \mathbb{R}^{n} \rightarrow \mathbb{R}^{n \times r}$ are locally Lipschitzian and with the following initial values

$$
p(0)=0, q(0)=0
$$

Definition 2.1. ${ }^{[34]}$ The solution process $\{x(t), t \geq 0\}$ of the stochastic system (3) is said to be bounded in probability if $\lim _{t \rightarrow \infty} \sup _{0 \leq c \leq \infty} P\{|x(t)|>c\}=0$ 
Definition 2.2. ${ }^{[11]}$ For any given $V(x) \in \mathscr{C}^{1,2}$, associated with the stochastic differential equation (3), the differential operator $\mathscr{L}$ can be defined as follows:

$$
\mathscr{L} V=\frac{\partial V}{\partial x} p(x)+\frac{1}{2} \operatorname{Tr}\left\{q^{T}(x) \frac{\partial^{2} V}{\partial x^{2}} q(x)\right\}
$$

Moreover, recalling the lemma ${ }^{[2]}$ below for the sufficient conditions of boundedness in the probabilistic sense.

Lemma 2.3. [2] Consider system (3) and suppose that there exists a positive-define and radially unbounded function $V(x) \in \mathscr{C}^{1,2}, \mu_{1}(\cdot), \mu_{2}(\cdot) \in \mathscr{K}_{\infty}$, positive-define and radially unbounded function $W(x)$ and constant $\bar{\gamma}>0$ such that

$$
\begin{aligned}
& \mu_{1}(|x|) \leq V(x) \leq \mu_{2}(|x|) \\
& \mathscr{L} V(x) \leq-W(x)+\bar{\gamma}
\end{aligned}
$$

then the solution process of the system (3) is bounded in the probabilistic sense.

\subsection{Entropy and Kernel Density Estima- tion}

The information theory ${ }^{[35]}$ has been introduced, where entropy can be used as a measure of the uncertainty of the random variables. For various purposes, a lot of different definitions of the entropy have been presented such as Shannon entropy, Rényi's entropy and Hartley entropy, etc. Without loss of the generality, the quadratic Rényi's entropy ${ }^{[36]}$ has been adopted in this paper.

$$
H_{2}(\bar{y})=-\log \int \gamma^{2}(\bar{y}) d \bar{y}
$$

where $\gamma(\cdot)$ denotes the joint probability density functions (JPDF) of the system outputs while multidimensional kernel density estimation (MKDE) ${ }^{[28]}$ can be used to approximate the JPDF of the random variables based on the collected sampling data. Notice that entropy of the Gaussian random variable is equal to its variance ${ }^{[37]}$.

For system outputs $\bar{y} \in \mathbb{R}^{n}$, denoting its sampling data as $\left\{\bar{y}_{k}: k=1, \ldots, N\right\}$, the probability density function of the system output $\bar{y}$ can be obtained approximately as follows:

$$
\hat{\gamma}(\bar{y})=\frac{1}{N} \sum_{k=1}^{N} G_{\Sigma}\left(\bar{y}-\bar{y}_{k}\right)
$$

where $G_{\Sigma}(\cdot)$ is the Gaussian function with the pre-specified covariance matrix $\Sigma$. Particularly, it has been defined as follows:

$$
G_{\Sigma}(x)=(2 \pi)^{-\frac{n}{2}}(\operatorname{det} \Sigma)^{-\frac{1}{2}} \exp \left(-\frac{1}{2} x^{T} \Sigma^{-1} x\right)
$$

Since the JPDF can be approximated vis data-based MKDE, Eq.(7) can be further rewritten as follows:

$$
H_{2}(\bar{y})=-\log V(\bar{y})
$$

where $V(\cdot)$ is information potential ${ }^{[35]}$. Furthermore, it can also be estimated using the sampling data as

$$
\hat{V}(\bar{y})=\frac{1}{N^{2}} \sum_{i, j=1}^{N} G_{\sqrt{2} \Sigma}\left(\bar{y}_{i}-\bar{y}_{j}\right)
$$

\subsection{Decoupling in probability sense}

For a MIMO dynamic stochastic system, the couplings among the system outputs are investigated by decoupling control based on the deterministic system model, however the analysis cannot reflect the full information of the randomness of the system output. Motivated by this shortcoming, the probabilistic decoupling has been presented in $2015^{[13]}$. In particular, the system outputs can be considered as stochastic processes in terms of time while the couplings can be represented by the independence of the random processes.

Using the probability density function (PDF) and joint probability density function (JPDF), the following definition is obtained.

Definition 2.4. For a multivariate dynamic stochastic system with n-dimensional system output, there exists a positive real stochastic coupling coefficient

$$
C_{S C}(y, t)=\left\|\gamma_{J}(y, t)-\prod_{i=1}^{n} \gamma\left(y_{i}, t\right)\right\|
$$

to describe the probabilistic couplings of the system outputs where $\gamma_{J}(y, t)$ denotes the JPDF of the multi-dimensional system output $y$. Moreover, the outputs of the multivariate dynamic stochastic systems are decoupled in the probabilistic sense if

$$
\lim _{t \rightarrow \infty} P\left\{C_{S C}(y, t) \geq \varepsilon\right\}=0
$$

for any small positive real constant $\varepsilon>0$

Based on this definition, a weak solution can also be defined by

Definition 2.5. A multiple input multiple output (MIMO) stochastic system with n-dimensional output is said to be decoupled in probability sense, if for any positive real constant $\varepsilon>0$ there exists a positve real constant $\delta$ such that the system output $y_{i}$ satisfy the following condition when $k>\delta$.

$$
\left\|\gamma_{J}(y, t)-\prod_{i=1}^{n} \gamma\left(y_{i}, t\right)\right\|<\varepsilon
$$

where $\|\cdot\|$ denotes the norm of the functions.

From the view of optimisation, the definition can be transformed as a cost function directly as follows:

$$
\min _{\Omega}\left\|\gamma_{J}(y, t)-\prod_{i=1}^{n} \gamma\left(y_{i}, t\right)\right\|
$$

In other words, the coupling attenuation can be achieved if the mentioned optimisation can be implemented while the performance of the system has been enhanced from the view of the system output randomness. Notice that the probabilistic decoupling is an extension of the traditional decoupling design, thus the benefits of the traditional decoupling design can be naturally inherited to the decoupling in the probabilistic sense. 


\section{Output Feedback Stabilisation}

\subsection{Linear estimator}

To achieve the ouput feedback, at first, the linear estimator can be designed as

$$
\begin{aligned}
d \hat{\bar{x}}_{i} & =\left(A_{i} \hat{\bar{x}}_{i}+\hat{\bar{x}}_{i+1}+L_{i}\left(\bar{y}-\hat{\bar{x}}_{1}\right)\right) d t, i=1, \cdots, m-1 \\
d \hat{\bar{x}}_{m} & =\left(A_{m} \hat{\bar{x}}_{m}+\bar{u}+L_{m}\left(\bar{y}-\hat{\bar{x}}_{1}\right)\right) d t
\end{aligned}
$$

where $L_{i}$ is the gain of estimator.

Defining the estimation error as $\tilde{\bar{x}}=\bar{x}-\hat{\bar{x}}$ which results in

$$
\begin{aligned}
d \tilde{\bar{x}} & =\left[\begin{array}{cccc}
A_{1}-L_{1} & I & & \\
-L_{2} & A_{2} & \ddots & \\
\vdots & \vdots & \ddots & I \\
-L_{m} & 0 & \ldots & A_{m}
\end{array}\right] \tilde{\bar{x}} d t+\left[\begin{array}{c}
G_{1}(\bar{y}) \\
G_{2}(\bar{y}) \\
\vdots \\
G_{m}(\bar{y})
\end{array}\right] d \beta_{t} \\
& =A_{0} \tilde{\bar{x}} d t+G_{0}(\bar{y}) d \beta_{t}
\end{aligned}
$$

Based upon the linear observer design method, $A_{0}$ should be adjusted to be Hurwitz, thus the investigated semi-linear stochastic system model with linear estimator can be rewritten as follows:

$$
\begin{aligned}
d \tilde{\bar{x}} & =A_{0} \tilde{\bar{x}} d t+G_{0}(\bar{y}) d \beta_{t} \\
d \bar{x}_{1} & =\left(A_{1} \bar{x}_{1}+\tilde{\bar{x}}_{2}+\hat{\bar{x}}_{2}\right) d t+G_{1}(\bar{y}) d \beta_{t} \\
d \hat{\bar{x}}_{i} & =\left(F_{i}+\hat{\bar{x}}_{i+1}\right) d t, i=2, \cdots, m-1 \\
d \hat{\bar{x}}_{m} & =\left(F_{m}+\bar{u}\right) d t \\
\bar{y} & =\bar{x}_{1}
\end{aligned}
$$

where $F_{i}=A_{i} \hat{\bar{x}}_{i}+L_{i}\left(\bar{y}-\hat{\bar{x}}_{1}\right)$ and $i=2, \cdots, m$.

Remark 2. To estimate the states of the system, existing observation methods can be used such as high-gain observer [38] which results in the extensions from various complex system descriptions.

\subsection{Block backstepping controller}

Notice that the semi-linear stochastic system with estimator is still in the strict-feedback format, block backstepping design can be used for stochastic system stabilization.

For $i$-th block of the investigated system(1), denote $\bar{\varphi}_{i}\left(\bar{y}, \hat{x}_{i}\right)$ as the virtual input. In particular, it can be reexpressed with elements as

$$
\bar{\varphi}_{i}\left(\bar{y}, \hat{x}_{i}\right)=\left[\varphi_{i 1}\left(\bar{y}, \hat{x}_{i}\right), \ldots, \varphi_{i n}\left(\bar{y}, \hat{x}_{i}\right)\right]^{T}
$$

where $\hat{x}_{i}=\left[\bar{x}_{1}, \hat{\bar{x}}_{2}, \ldots, \hat{\bar{x}}_{i}\right]$ and $i=1, \cdots, m-1$.

In particular, the following equation can be obtained for the first step following Itô's lemma, Eq.(22).

$$
\begin{aligned}
d \bar{z}_{1} & =d \hat{\bar{x}}_{2}-d \bar{\varphi}_{1}(\bar{y}) \\
& =\left(F_{2}-\Phi_{1}\left(A_{1} \bar{x}_{1}+\tilde{\bar{x}}_{2}+\hat{\bar{x}}_{2}\right)-\frac{1}{2} \Pi_{1}+\hat{\bar{x}}_{3}\right) \\
& -\Phi_{1} G_{1}(\bar{y}) d \beta_{t}
\end{aligned}
$$

where

$$
\begin{aligned}
\Phi_{1} & =\left[\nabla_{\bar{y}} \varphi_{11}(\bar{y}), \ldots, \nabla_{\bar{y}} \varphi_{1 n}(\bar{y})\right]^{T} \\
\Pi_{1}= & {\left[\begin{array}{l}
\operatorname{Tr}\left\{G_{1}^{T}(\bar{x})\left(H_{\bar{x}} \varphi_{11}\left(\bar{x}_{1}\right)\right) G_{1}\left(\bar{x}_{1}\right)\right\} \\
\vdots \\
\operatorname{Tr}\left\{G_{1}^{T}(\bar{x})\left(H_{\bar{x}} \varphi_{1 n}\left(\bar{x}_{1}\right)\right) G_{1}\left(\bar{x}_{1}\right)\right\}
\end{array}\right] }
\end{aligned}
$$

Based upon the equation above, the virtual input for the next step can be obtained recursively and the error variables can be formulated as follows:

$$
\bar{z}_{i}=\hat{\bar{x}}_{i+1}-\bar{\varphi}_{i}\left(\bar{y}, \hat{x}_{i}\right)
$$

where

$$
\bar{z}_{i}=\left[z_{i 1}, \ldots, z_{i n}\right]^{T}, i=1, \cdots, m-1
$$

Based on Itô's lemma, we have

$$
\begin{aligned}
d \bar{z}_{i} & =\left[\left(F_{i+1}+\hat{\bar{x}}_{i+2}\right)-\Phi_{1}\left(F_{1}+\hat{\bar{x}}_{2}\right)-\frac{1}{2} \Pi_{1}\right. \\
& \left.-\sum_{l=2}^{i} \Phi_{l}\left(F_{l}+\hat{\bar{x}}_{l+1}\right)\right] d t-\Phi_{1} G_{1}(\bar{y}) d \beta_{t} \\
& =\left(\Xi_{i}+\hat{\bar{x}}_{i+2}\right) d t-\Phi_{1} G_{1}(\bar{y}) d \beta_{t}
\end{aligned}
$$

while

$$
\begin{aligned}
& \Phi_{l}=\left[\nabla_{\hat{\bar{x}}_{l}} \varphi_{l 1}\left(\bar{y}, \hat{x}_{l}\right), \ldots, \nabla_{\hat{\bar{x}}_{l}} \varphi_{l n}\left(\bar{y}, \hat{x}_{l}\right)\right]^{T} \\
& \Xi_{i}=F_{i+1}-\Phi_{1}\left(F_{1}+\hat{\bar{x}}_{2}\right)-\frac{1}{2} \Pi_{1}-\sum_{l=2}^{i} \Phi_{l}\left(F_{l}+\hat{\bar{x}}_{l+1}\right)
\end{aligned}
$$

To stabilize the closed-loop stochastic system, a Lyapunov function candidate has been considered for backstepping design.

$$
\bar{V}=\frac{1}{2} \sum_{k=1}^{n} \bar{y}_{k}^{2}+\frac{b}{2}\left(\tilde{x}^{T} \bar{P} \tilde{x}\right)^{2}+\frac{1}{4} \sum_{i=1}^{m-1} \sum_{l=1}^{n} \bar{z}_{i l}^{4}
$$

where $P$ stands for the positive definite matrix and $A_{0}{ }^{T} \bar{P}+$ $\bar{P} A_{0}<0$.

Using the property of $\mathscr{L} \bar{V}$ and the presented Lyapunov function candidate, we have

$$
\begin{aligned}
\mathscr{L} \bar{V} & =\bar{y}^{T}\left(A_{1} \bar{y}+\tilde{\bar{x}}_{2}+\hat{\bar{x}}_{2}\right)+\frac{1}{2} \operatorname{Tr}\left\{G_{1}^{T}(\bar{y}) G_{1}(\bar{y})\right\} \\
& +\sum_{i=1}^{m-1} \eta_{i}\left(\Xi_{i}+\hat{\bar{x}}_{i+2}\right)+\frac{3}{2} \operatorname{Tr}\left\{\Gamma_{i} \Phi_{1} G_{1}(\bar{y})\left(\Phi_{1} G_{1}(\bar{y})\right)^{T}\right\} \\
& +2 b \operatorname{Tr}\left\{G_{0}^{T}(\bar{y})\left(2 \bar{P} \tilde{x} \tilde{x}^{T} \bar{P}+\tilde{x}^{T} \bar{P} \tilde{x} \bar{P}\right) G_{0}(\bar{y})\right\} \\
& -b \tilde{x}^{T} \bar{P} \tilde{x}\|\tilde{x}\|^{2}
\end{aligned}
$$

where

$$
\begin{aligned}
\eta_{i} & =\left[z_{i 1}^{3}, \ldots, z_{i n}^{3}\right] \\
\Gamma_{i} & =\operatorname{diag}\left(z_{i 1}^{2}, \ldots, z_{i n}^{2}\right)
\end{aligned}
$$

Moreover, a useful lemma is given here to deal with the trace terms of $\mathscr{L} \bar{V}$. 
Lemma 3.1. Considering the square matrices $A_{1}, A_{2}, B \in$ $R^{n \times n}$ are of $n$-dimension and $D \in R^{n \times n}$ is a diagonal matrix, in particular, $A_{1}=\left[\bar{a}_{11}, \ldots, \bar{a}_{1 n}\right]^{T}, A_{2}=\left[\bar{a}_{21}, \ldots, \bar{a}_{2 n}\right]$ and $D=\operatorname{diag}\left\{d_{1}, \ldots, d_{n}\right\}$, such that, $\operatorname{Tr}\left\{D A_{1} B A_{2}\right\}$ is bounded.

$$
\operatorname{Tr}\left\{D A_{1} B A_{2}\right\} \leq \sum_{i=1}^{n}\left\|d_{i}\right\|\left\|\bar{a}_{1 i}\right\|\left\|\bar{a}_{2 i}\right\|\|B\|
$$

Proof. Using the structure of the matrices which has been mentioned above, we can have

$$
\begin{aligned}
& \operatorname{Tr}\left\{D A_{1} B A_{2}\right\} \\
& =\operatorname{Tr}\left\{\left[\begin{array}{ccc}
d_{1} & & \\
& \ddots & \\
& & d_{n}
\end{array}\right]\left[\begin{array}{c}
a_{11} \\
\vdots \\
a_{1 n}
\end{array}\right] B\left[\begin{array}{lll}
a_{21} & \cdots & a_{2 n}
\end{array}\right]\right\} \\
& =\operatorname{Tr}\left\{\left[\begin{array}{ccc}
d_{1} & & \\
& \ddots & \\
& & d_{n}
\end{array}\right]\left[\begin{array}{ccc}
a_{11} B a_{21} & \ldots & a_{11} B a_{2 n} \\
\vdots & \ddots & \vdots \\
a_{1 n} B a_{21} & \ldots & a_{1 n} B a_{2 n}
\end{array}\right]\right\} \\
& =\sum_{i=1}^{n} d_{i} a_{1 i} B a_{2 i} \\
& \leq \sum_{i=1}^{n}\left\|d_{i} a_{1 i} B a_{2 i}\right\|
\end{aligned}
$$

Using the norm operation, the following inequality can be obtained

$$
\sum_{i=1}^{n}\left\|d_{i} a_{1 i} B a_{2 i}\right\| \leq \sum_{i=1}^{n}\left\|d_{i}\right\|\left\|a_{1 i}\right\|\left\|a_{2 i}\right\|\|B\|
$$

which completes the proof.

Using Lemma 3.1 repeatedly, the following inequalities can be obtained to simplify the trace terms in Eq.(26) based upon Young's inequality.

Firstly,

$$
\begin{aligned}
\operatorname{Tr} & \left\{\Gamma \Phi_{1} G_{1}(\bar{y}) G_{1}^{T}(\bar{y}) \Phi_{1}^{T}\right\} \\
& \leq \sum_{i=1}^{n} z_{1 i}^{2}\left\|\nabla_{\bar{x}} \varphi_{1 i}^{T}(\bar{y})\right\|_{2}\left\|\nabla_{\bar{x}} \varphi_{1 i}(\bar{y})\right\|_{2}\left\|G_{1}(\bar{y})\right\|_{2}\left\|G_{1}^{T}(\bar{y})\right\|_{2} \\
& \leq \sum_{i=1}^{n} \frac{\varepsilon_{1 i}^{2}}{2} z_{1 i}^{4}\left\|\nabla_{\bar{x}} \varphi_{1 i}^{T}(\bar{y})\right\|^{2}+\sum_{i=1}^{n} \frac{1}{2 \varepsilon_{1 i}^{2}}\left\|G_{1}(\bar{y})\right\|_{2}^{4} \\
& =\sum_{i=1}^{n} \frac{\varepsilon_{1 i}^{2}}{2} z_{1 i}^{4}\left\|\nabla_{\bar{x}} \varphi_{1 i}^{T}\left(\bar{x}_{1}\right)\right\|^{2}+\sum_{i=1}^{n} \frac{1}{2 \varepsilon_{1 i}^{2}} \sigma_{1}^{4}
\end{aligned}
$$

Next, the following inequalities are also obtained.

$$
\operatorname{Tr}\left\{G_{1}^{T}(\bar{y}) G_{1}(\bar{y})\right\} \leq n\left\|G_{1}(\bar{y})\right\|^{2}=n \sigma_{1}^{2}
$$

and

$$
\begin{aligned}
2 b T r & \left\{G_{0}^{T}(\bar{y})\left(2 \bar{P} \tilde{x} \tilde{x}^{T} \bar{P}+\tilde{x}^{T} \bar{P} \tilde{x} \bar{P}\right) G_{0}(\bar{y})\right\} \\
& =4 b\left\|G_{0}^{T}(\bar{y}) \bar{P} \tilde{x}\right\|_{F}^{2}+2 b \tilde{x} \bar{P}^{T} \tilde{x} T r\left\{\bar{P} G_{0}(\bar{y}) G_{0}^{T}(\bar{y})\right\} \\
& \leq 4 b \sqrt{s}\left\|G_{0}^{T}(\bar{y})\right\|^{2}\|\bar{P}\|^{2}\|\tilde{x}\|^{2} \\
& +2 b\left\|\operatorname{Tr}\left\{\bar{P} G_{0}(\bar{y}) G_{0}^{T}(\bar{y})\right\}\right\|\|\bar{P}\|\|\tilde{x}\|^{2} \\
& \leq\left(\frac{\tilde{\varepsilon}_{1}^{2}+\tilde{\varepsilon}_{2}^{2}}{2}\right)\|\tilde{x}\|^{4}+\frac{8 b^{2} s}{\tilde{\varepsilon}_{1}^{2}}\left\|G_{0}^{T}(\bar{y})\right\|^{4}\|\bar{P}\|^{4} \\
& +\frac{2 b^{2}}{\tilde{\varepsilon}_{2}^{2}}\|\bar{P}\|^{2}\left(\sum_{i=1}^{n m}\left|p_{i}\right|\left\|G_{0}^{T}(\bar{y})\right\|^{2}\right)^{2} \\
& =\left(\frac{\tilde{\varepsilon}_{1}^{2}+\tilde{\varepsilon}_{2}^{2}}{2}\right)\|\tilde{x}\|^{4}+\tilde{c}
\end{aligned}
$$

where $\tilde{\varepsilon}_{1}, \tilde{\varepsilon}_{2}, \varepsilon_{1 i}$ stand for specified real positive numbers and $\|\cdot\|_{F}$ denotes the Frobenius norm.

Moreover, as $\tilde{c} \geq 0$, we have

$\tilde{c}=\frac{8 b^{2} s}{\tilde{\varepsilon}_{1}^{2}}\left\|G_{0}^{T}(\bar{y})\right\|^{4}\|\bar{P}\|^{4}+\frac{2 b^{2}}{\tilde{\varepsilon}_{2}^{2}}\|\bar{P}\|^{2}\left(\sum_{i=1}^{n m}\left|p_{i}\right|\left\|G_{0}^{T}(\bar{y})\right\|^{2}\right)^{2}$

and

$$
-b \tilde{x}^{T} \bar{P} \tilde{x}\|\tilde{x}\|^{2} \leq-b \lambda_{\min }\{\bar{P}\}\|\tilde{x}\|^{4}
$$

Substituting Eq. (23)-Eq. (26) to $\mathscr{L} \bar{V}$, Eq. (26) can be rewritten as a result. Particularly, the following inequality can be further given to illustrate the property of the controller for system stabilization.

$$
\begin{aligned}
& \mathscr{L} \bar{V} \leq \bar{y}^{T}\left(A_{1} \bar{y}+\tilde{\bar{x}}_{2}+\hat{\bar{x}}_{2}\right)+\frac{1}{2} n \sigma_{1}^{2}+\sum_{i=1}^{m-1} \eta_{i}\left(\Xi_{i}+\hat{\bar{x}}_{i+2}\right) \\
& +\frac{3}{2}\left(\sum_{i=1}^{n} \frac{\varepsilon_{1 i}^{2}}{2} z_{1 i}^{4}\left\|\nabla_{\bar{x}} \varphi_{1 i}^{T}\left(\bar{x}_{1}\right)\right\|^{2}+\sum_{i=1}^{n} \frac{1}{2 \varepsilon_{1 i}^{2}} \sigma_{1}^{4}\right) \\
& -b \lambda_{\min }\{\bar{P}\}\|\tilde{x}\|^{4}+\left(\frac{\tilde{\varepsilon}_{1}^{2}+\tilde{\varepsilon}_{2}^{2}}{2}\right)\|\tilde{x}\|^{4}+\tilde{c} \\
& =\bar{y}^{T}\left(A_{1} \bar{y}+\tilde{\bar{x}}_{2}+\hat{\bar{x}}_{2}\right) \\
& +\sum_{i=2}^{m-1} \eta_{i}\left(\Xi_{i}+\bar{z}_{i+1}+\bar{\varphi}_{i+1}\left(\bar{y}, \hat{x}_{i+1}\right)\right) \\
& +\eta_{1}\left(\Xi_{1}+\bar{z}_{2}+\bar{\varphi}_{2}\left(\bar{y}, \hat{\bar{x}} x_{2}\right)+\left[\begin{array}{c}
\frac{\varepsilon_{11}^{2}}{2} z_{11}\left\|\nabla_{\bar{x}} \varphi_{11}^{T}(\bar{y})\right\|^{2} \\
\vdots \\
\frac{\varepsilon_{1 n}^{2}}{2} z_{1 n}\left\|\nabla_{\bar{x}} \varphi_{1 n}^{T}(\bar{y})\right\|^{2}
\end{array}\right]\right) \\
& +\frac{3}{2} \sum_{i=1}^{n} \frac{1}{2 \varepsilon_{1 i}^{2}} \sigma_{1}^{4}+\tilde{c}-\left(b \lambda_{\min }\{\bar{P}\}-\frac{\tilde{\varepsilon}_{1}^{2}+\tilde{\varepsilon}_{2}^{2}}{2}\right)\|\tilde{x}\|^{4}
\end{aligned}
$$

Motivated by Lemma 1, the virtual inputs and control input can be designed as follows:

$$
\begin{aligned}
\bar{\varphi}_{1}(\bar{y}) & =\left(-W-A_{1}\right) \bar{y}-\tilde{\bar{x}}_{2} \\
\bar{\varphi}_{2}\left(\bar{y}, \hat{\bar{x}}_{2}\right) & =-\Xi_{1}-\bar{z}_{2}-\Lambda-C_{1} \bar{z}_{1} \\
\bar{\varphi}_{i+1}\left(\bar{y}, \hat{x}_{i+1}\right) & =-\Xi_{i}-\bar{z}_{i+1}-C_{i} \bar{z}_{i} \\
u=\bar{\varphi}_{m}\left(\bar{y}, \hat{x}_{m}\right) & =-\Xi_{m-1}-\bar{z}_{m}-C_{m-1} \bar{z}_{m-1}
\end{aligned}
$$


where $W$ is a positive definite matrix.

$$
\begin{gathered}
\Lambda=\left[\begin{array}{c}
\frac{\varepsilon_{11}^{2}}{2} z_{11}\left\|\nabla_{\bar{x}} \varphi_{11}^{T}(\bar{y})\right\|^{2} \\
\vdots \\
\frac{\varepsilon_{1 n}^{2}}{2} z_{1 n}\left\|\nabla_{\bar{x}} \varphi_{1 n}^{T}(\bar{y})\right\|^{2}
\end{array}\right] \\
C_{i}=\operatorname{diag}\left[c_{i 1}, \ldots, c_{i n}\right], c_{i j}>0
\end{gathered}
$$

Furthermore, $\mathscr{L} \bar{V}$ can be re-expressed as

$$
\mathscr{L} \bar{V}=-\bar{y}^{T} W \bar{y}-\tilde{p}\|\tilde{x}\|^{4}-\sum_{i=1}^{m-1} \sum_{l=1}^{n} c_{i l} \bar{z}_{i l}^{4}+\bar{c}
$$

while

$$
\tilde{p}=b \lambda_{\min }\{P\}-\frac{\tilde{\varepsilon}_{1}^{2}+\tilde{\varepsilon}_{2}^{2}}{2}, \bar{c}=\frac{3}{2} \sum_{i=1}^{n} \frac{1}{2 \varepsilon_{1 i}^{2}} \sigma_{1}^{4}+\tilde{c}
$$

Therefore, the following theorem can be summarised for the stabilisation problem of the investigated closed-loop semi-linear stochastic system.

Theorem 3.2. Using the linear estimator (16) and control law (37), the system outputs of the semi-linear stochastic system (1) are bounded in the probabilistic sense if there exists a positive definite matrix $\bar{P}$ satisfying the conditions: $\tilde{p}>0$ and $A_{0}{ }^{T} \bar{P}+\bar{P} A_{0}<0$.

Proof. Notice that $A_{0}, P$ and $\tilde{p}$ have been shown in Eqs. (17), (25) and (40), the proof has been completed following the analysis obtained above.

\section{Output Transient Optimization}

The parametric optimisation can be further taken into account following the criterion in order to attenuate the randomness and probabilistic coupling respectively. Motivated by stochastic distribution optimisation ${ }^{[39]}$, the distribution information will be adopted. To implement the optimisation operation, the transient process should be sampled where $k$ is the sampling index.

\subsection{Output randomness attenuation}

Following the discussion in section 2 , in order to describe the randomness of the system outputs, the performance criterion can be given using the concept of the entropy.

$$
J_{k}=-\log \hat{V}_{k}\left(\bar{y}, W_{0}\right)
$$

where $\hat{V}_{k}\left(\bar{y}, W_{0}\right)$ stands for the estimated information potential of $\bar{y}, \mathrm{~W}_{0}=\left\{W, \tilde{\varepsilon}_{1}, \tilde{\varepsilon}_{2}, \varepsilon_{1 i}, C_{i}\right\}, i=1, \ldots, n$ has been defined as the design parametric set while $k$ is the sampling index. Note that the $\log (\cdot)$ function is a monotonic increasing function, maximizing the information potential can be used to take the place of minimizing of the entropy, then the performance criterion can be simplified only using the information potential $\hat{V}\left(\bar{y}, W_{0}\right)$.

Furthermore, another theorem is given to state that the presented performance criterion is globally convex, if another assumption is added as follows.

Assumption 2. The vector-valued stochastic system output $\bar{y}$ satisfies the following inequality:

$$
\frac{\partial \bar{y}}{\partial W_{0}} \leq \bar{M}
$$

where the real positive matrix $\bar{M}$ denotes the upper bound.

Theorem 4.1. Based upon the presented control algorithm, there exists a real positive number $\delta_{0}>0$, such that the information potential is globally concave in terms of the design parameter $W_{0}$ for all $\lambda_{\min }(\Sigma)>\delta_{0}$. It implies that the equivalent performance criterion (41) is globally convex and the optimum exists.

Proof. Denote $\varepsilon_{i j, k}=\bar{y}_{i, k}-\bar{y}_{j, k}$, then we have

$$
\begin{aligned}
& \frac{\partial^{2} \hat{V}_{k}\left(W_{0}\right)}{\partial W_{0}^{2}}=\frac{1}{N^{2}} \frac{\partial}{\partial W_{0}} \sum_{i, j=1}^{N} \frac{\partial}{\partial W_{0}} G_{\sqrt{2} \Sigma}\left(\varepsilon_{i j, k}\right) \\
& =\frac{1}{N^{2}} \frac{\partial}{\partial W_{0}} \sum_{i, j=1}^{N} \frac{\partial G_{\sqrt{2} \Sigma}\left(\varepsilon_{i j, k}\right)}{\partial \varepsilon_{i j, k}} \frac{\partial \varepsilon_{i j, k}}{\partial W_{0}} \\
& \leq-\frac{1}{N^{2}}(\sqrt{2} \Sigma)^{-1} \frac{\partial}{\partial W_{0}} \sum_{i, j=1}^{N} G_{\sqrt{2} \Sigma}\left(\varepsilon_{i j, k}\right) \times \varepsilon_{i j, k} \bar{M} \\
& =-\frac{\bar{M}}{N^{2}}(\sqrt{2} \Sigma)^{-1} \sum_{i, j=1}^{N} G_{\sqrt{2} \Sigma}\left(\varepsilon_{i j, k}\right) \\
& \times\left(\varepsilon_{i j, k}^{T}\left(\bar{M}-(\sqrt{2} \Sigma)^{-1}\right) \varepsilon_{i j, k}\right)
\end{aligned}
$$

Note that $\frac{\partial^{2} V_{k}(\varepsilon)}{\partial W_{0}^{2}} \leq 0$ if $\bar{M} \geq(\sqrt{2} \Sigma)^{-1}$. It implies that the eigenvalues of $\frac{\partial^{2} V_{k}(\varepsilon)}{\partial W_{0}^{2}}$ approach $0^{-}$as $\lambda_{\min }(\Sigma)$ goes to infinity. Using the Lemma 3 in ${ }^{[40]}, \hat{V}_{k}\left(\bar{y}, W_{0}\right)$ is a concave function once $\lambda_{\min }(\Sigma)$ is sufficiently large. Thus, the performance criterion (41) is a convex function which results in the global optimum. It ends the proof of this theorem.

Once the performance criterion is proved to be a convex function, the standard convex optimization approach, e.g. gradient descent optimization, can be adopted directly. In particular, we have

$$
W_{0, k+1}=W_{0, k}-\left.\epsilon_{1} \frac{\partial \hat{V}_{k}\left(W_{0}\right)}{\partial W_{0}}\right|_{W_{0}=W_{0, k}}
$$

where $\epsilon_{1}>0$ stands for the pre-specified searching rate and the information potential of each system outputs which can be approximated by MKDE.

\subsection{Output probabilistic coupling attenu- ation}

Notice that decoupling design is an important topic for multi-variable control systems, then the couplings among the system outputs can also be attenuated based on the presented framework. Motivated by the concept of probabilistic decoupling, the performance criterion can be described using mutual information (MI) of the system outputs ${ }^{[41]}$.

Combining Cauchy-Schwarz mutual information (CSMI) and quadratic Rényi entropy, the multi-variable CSMI is expressed as follows:

$$
I_{C S}(\bar{y})=-\log \frac{\left(\int \gamma(\bar{y}) \prod_{i=1}^{n} \gamma_{i}\left(y_{i}\right) d y\right)^{2}}{\left(\int \gamma(\bar{y}) d y\right)\left(\int \prod_{i=1}^{n} \gamma_{i}^{2}\left(y_{i}\right) d y\right)}
$$


Notice that $I_{c s}(\bar{y}) \geq 0$ with equality if and only if the system outputs are probabilistic independent. Furthermore, we have

$$
I_{C S}(\bar{y})=\log V_{J}+\log V_{M}-2 \log V_{C}
$$

where

$$
\begin{aligned}
V_{J} & =\int \gamma^{2}(\bar{y}) d y \\
V_{M} & =\int \prod_{i=1}^{n} \gamma_{i}^{2}\left(y_{i}\right) d y \\
V_{C} & =\int \gamma(\bar{y}) \prod_{i=1}^{n} \gamma_{i}\left(y_{i}\right) d y
\end{aligned}
$$

stand for information potentials. Compared with the definition of Rényi's entropy in section II, the value of the information potentials can be estimated by sampling data.

In particular, the kernel density estimation can also be used where the performance criterion mentioned above can be rewritten as

$$
I_{c s}(\bar{y})=\log \frac{\left(\frac{1}{N^{2}} \sum_{i=1}^{N} \sum_{j=1}^{N} \prod_{k=1}^{n} \hat{V}_{k}(i, j)\right) \prod_{k=1}^{n} \hat{V}_{k}}{\left(\frac{1}{N} \sum_{i=1}^{N} \prod_{k=1}^{n} \hat{V}_{k}(i)\right)}
$$

where

$$
\begin{aligned}
& \hat{V}_{k}(i)=\frac{1}{N} \sum_{j=1}^{N} \hat{V}_{k}(i, j) \\
& \hat{V}_{k}(i, j)=G_{\sqrt{2} \Sigma}\left(y_{k, i}-y_{k, j}\right) \\
& \hat{V}_{k}=\frac{1}{N} \sum_{j=1}^{N} \hat{V}_{k}(i), k=1,2, \ldots, n
\end{aligned}
$$

Thus, the performance criterion can be estimated for each sampling instant using the collected data.

$$
\begin{aligned}
I_{C S}(\bar{y}) & =\frac{1}{N^{2}} \sum_{i=1}^{N} \sum_{j=1}^{N} \prod_{k=1}^{n} \hat{V}_{k}(i, j) \\
& +\prod_{k=1}^{n} \hat{V}_{k}-\frac{2}{N} \sum_{i=1}^{N} \prod_{k=1}^{n} \hat{V}_{k}(i)
\end{aligned}
$$

Similar to the criterion (41) and the theorem 4.1, it can be shown that the mutual information criterion is convex based on similar assumptions to those in Theorem 4.1. Then the decoupling in the probabilistic sense can be achieved following the

$$
W_{0, k+1}=W_{0, k}-\left.\epsilon_{2} \frac{\partial I_{C S}\left(W_{0}\right)}{\partial W_{0}}\right|_{W_{0}=W_{0, k}}
$$

while $\epsilon_{2}>0$ stands for the pre-specified searching rate.

\subsection{Design Procedure}

To clearly illustrate progress of the presented control algorithm, one flow chart is shown here to clarify the design procedure.

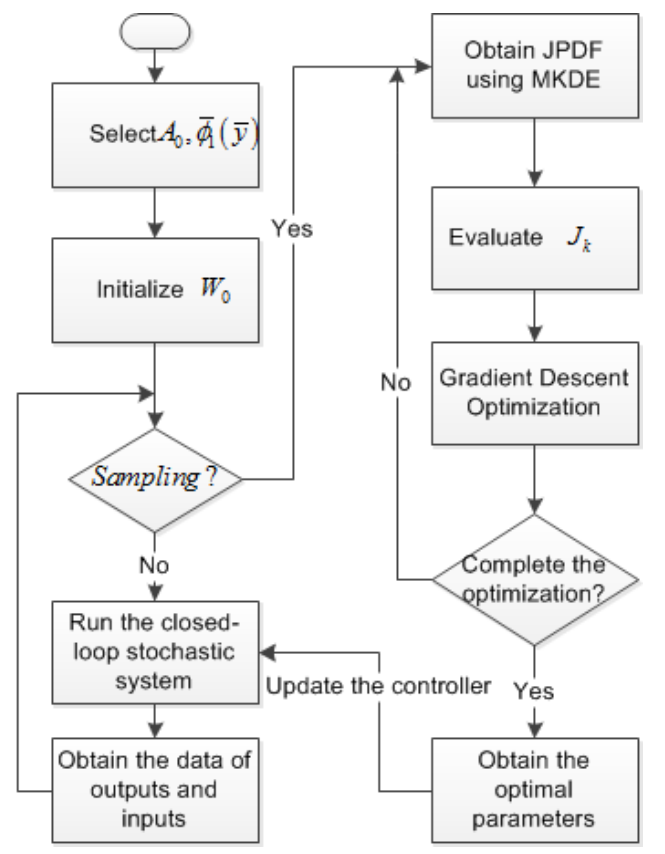

Fig. 1 The flowchart of the presented control framework design procedure

\section{A Numerical Example}

To demonstrate the presented algorithm procedure, a multi-variable semi-linear stochastic system is shown as follows:

$$
\begin{aligned}
d \bar{x}_{1} & =\left(\left[\begin{array}{cc}
-1 & 0.5 \\
0 & -2
\end{array}\right] \bar{x}_{1}+\bar{x}_{2}\right) d t+\sin \left(\bar{x}_{1}\right) d \beta_{t} \\
d \bar{x}_{2} & =\left(\left[\begin{array}{cc}
-1.5 & 0 \\
-0.5 & -1
\end{array}\right] \bar{x}_{2}+\bar{u}\right) d t+\cos \left(\bar{x}_{1}\right) d \beta_{t} \\
\bar{y} & =\bar{x}_{1}
\end{aligned}
$$

where the matrices $A_{1}, A_{2}, G_{1}$ and $G_{2}$ are obtained as the coefficient matrices.

In addition, the linear observer can be designed with the observation gain $L_{1}=L_{2}=\operatorname{diag}\{15,15\}$. Then, the entire system with linear estimator has been further rewritten as follows:

$$
\begin{aligned}
d \bar{y} & =\left(\left[\begin{array}{cc}
-1 & 0.5 \\
0 & -2
\end{array}\right] \bar{y}+\tilde{\bar{x}}_{2}+\hat{\bar{x}}_{2}\right) d t+\sin (\bar{y}) d \beta_{t} \\
d \hat{\bar{x}}_{2} & =\left(\left[\begin{array}{cc}
-1.5 & 0 \\
-0.5 & -1
\end{array}\right] \hat{\bar{x}}_{2}+\left[\begin{array}{cc}
2 & 0 \\
0 & 3
\end{array}\right] \tilde{\bar{x}}_{1}+\bar{u}\right) d t \\
d\left[\begin{array}{c}
\tilde{\bar{x}}_{1} \\
\tilde{\bar{x}}_{2}
\end{array}\right]= & {\left[\begin{array}{cccc}
-3 & 0.5 & 1 & 0 \\
0 & -5 & 0 & 1 \\
-2 & 0 & -1.5 & 0 \\
0 & -3 & -0.5 & -1
\end{array}\right]\left[\begin{array}{l}
\tilde{\bar{x}}_{1} \\
\tilde{\bar{x}}_{2}
\end{array}\right] d t } \\
+ & {\left[\begin{array}{c}
\sin (\bar{y}) \\
\cos (\bar{y})
\end{array}\right] d \beta_{t} }
\end{aligned}
$$

while the matrices $A_{0}$ and $G_{0}$ are obtained. Note that $A_{0}$ is Hurwitz. 
Following the discussion in section 3 , the control input and first virtual control input can be obtained by Eq. (37), which shows that the performance of the controller will be affected by the design parameters $W_{0}$. Based on the afore-mentioned optimisation operation, the optimal parameters can be searched with the pre-selected initial value $W_{0}=\operatorname{diag}\{-20,25\}$ and other parameters can also be prespecified randomly as positive numbers.

To set up the simulation, $k$ is designed as $0.01 \mathrm{~s}$ then Figs. 2-6 indicate the performance of the investigated closed-loop stochastic semi-linear system, especially for the transient performance of the system outputs. In particular, Fig. 2 shows the trajectories of the system outputs where all the system outputs are bounded in the probabilistic sense. Fig. 3 indicates the control inputs signal which is also bounded in the probabilistic sense while the entropybased performance criterion is given in Fig. 4. It has been shown that the transient randomness has been attenuated along the decrease of the performance criterion. In other words, $J$ descends by searching the optimal design parameters in control law. Note that the entropy is approximated by KDE. In addition, all the results shown above are based on the model where $G_{2}(0) \neq 0$. Alternatively, the system outputs will converge to 0 simultaneously in the probabilistic sense if $G_{2}(\bar{y})=\sin (\bar{y})$ which has been demonstrated by Fig. 5 .

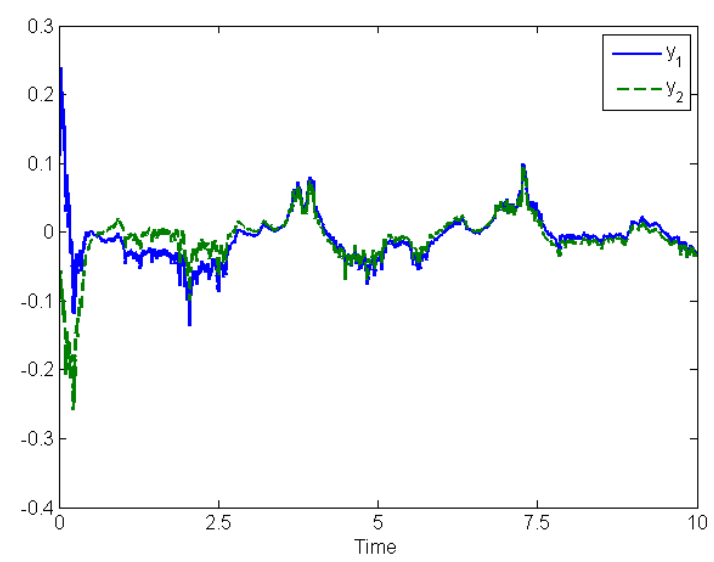

Fig. 2 System outputs' trajectories of the closed-loop semi-linear stochastic system.

Furthermore, the probabilistic decoupling problem can also be considered following the presented algorithm where $y_{1}$ and $y_{2}$ are coupled with each other transiently before the stabilisation of system output is achieved. To quantify the system output's coupling, the mutual information performance criterion can be used to replace the entropy-based criterion, then the following results are given where the mutual information of the system output has been minimised. It implies that the independence of the system outputs for transient performance is increasing.

Notice that $G_{2}(0) \neq 0$ and the value of the mutual information based performance criterion has been processed using sliding average mode, while the mutual information does not converge to zero due to the randomness of $\beta_{t}$. However, as an optimisation problem, the minimum is achieved

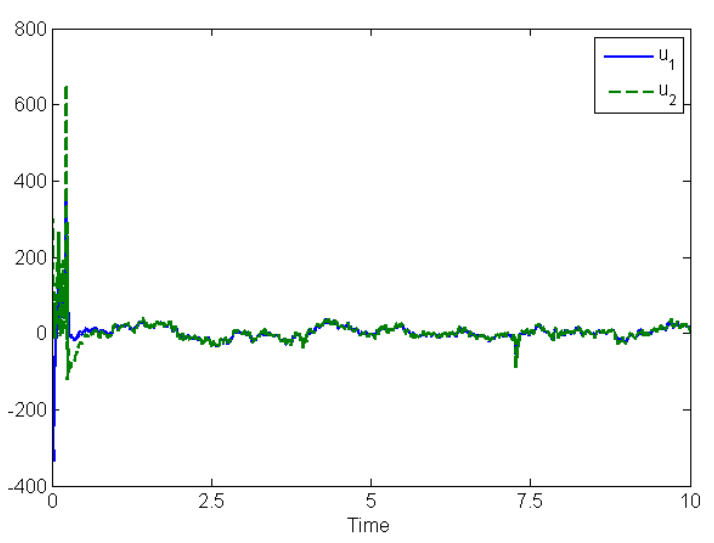

Fig. 3 The designed control inputs for the closed-loop semi-linear stochastic system.

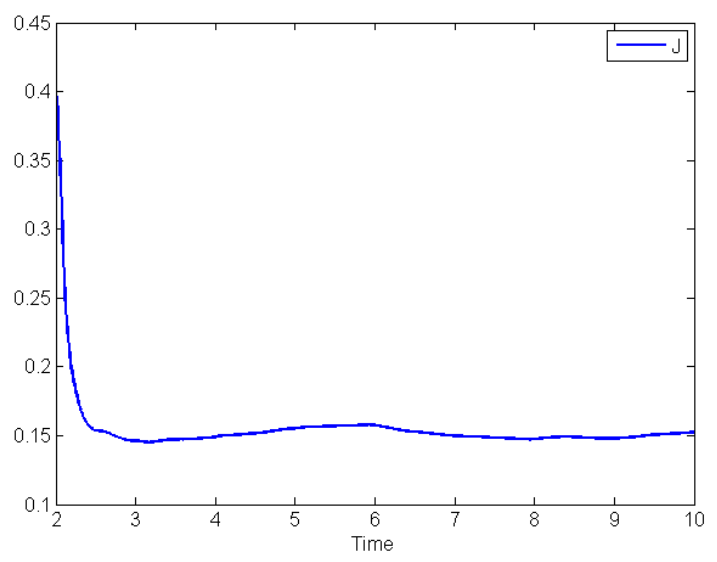

Fig. 4 The value of entropy-based performance criterion $J$ to attenuate the transient randomness of the system outputs

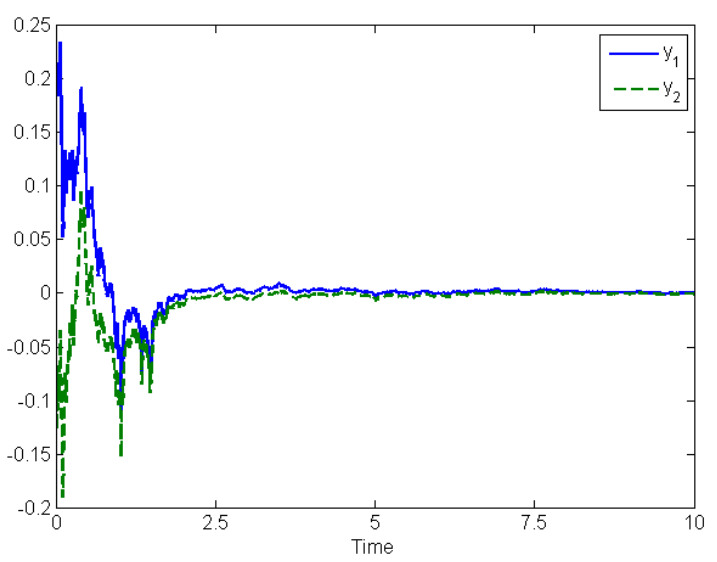

Fig. 5 System outputs' trajectories of the closed-loop semi-linear stochastic system with $G_{2}(0)=0$.

which also satisfied our design requirement for decoupling design in probability sense.

As an additional extension of the given numerical exam- 


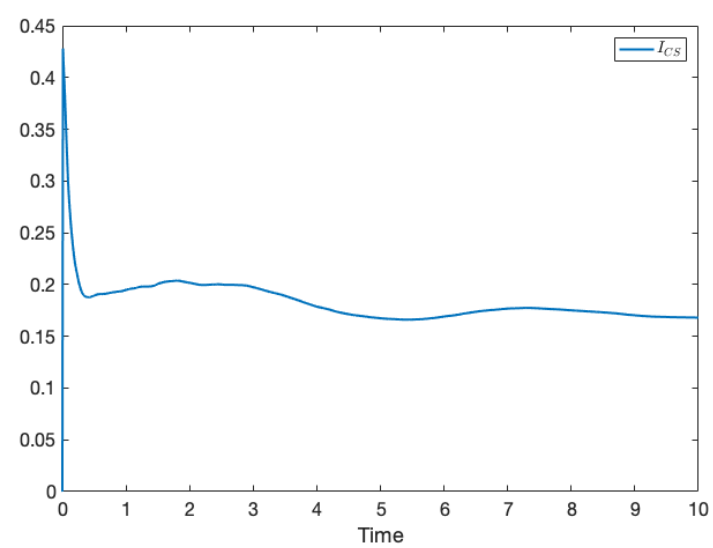

Fig. 6 The value of mutual information based performance criterion $I_{C S}$ to attenuate the transient probabilistic couplings of the system outputs.

ple, the system coefficient matrices can be replaced as follows to validate the performance of the presented algorithm for the system model with an unstable system dynamic matrix.

$$
A_{1}=\left[\begin{array}{cc}
-10 & 2 \\
-1 & 3
\end{array}\right], A_{2}=\left[\begin{array}{ll}
-5 & 1 \\
-2 & 4
\end{array}\right]
$$

where $A_{1}$ and $A_{2}$ are not Hurwitz. $G_{1}, G_{2}$ and initial values are given as same as the example above. Thus, the performances of the system output and control input are shown by Figs. 7 and 8 with the presented controller design. In particular, the system outputs are stabilised and converge to zero in probability one, meanwhile the control inputs converge to zero once the system outputs are stabilised. In addition, the transient randomness has been attenuated following the minimum entropy criterion.

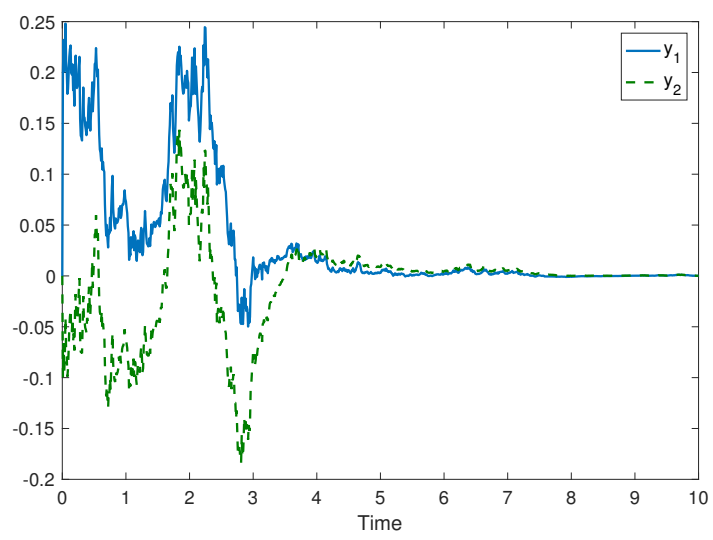

Fig. 7 System outputs' trajectories of the closed-loop semi-linear stochastic system with unstable $A$ matrix.

To highlight the power of the presented control algorithm, the performances are also compared to widely used PI design for the system model (53), where $K p=$ $\operatorname{diag}\{10,14\}$ and $K i=\operatorname{diag}\{0.05,-0.01\}$. Note that the

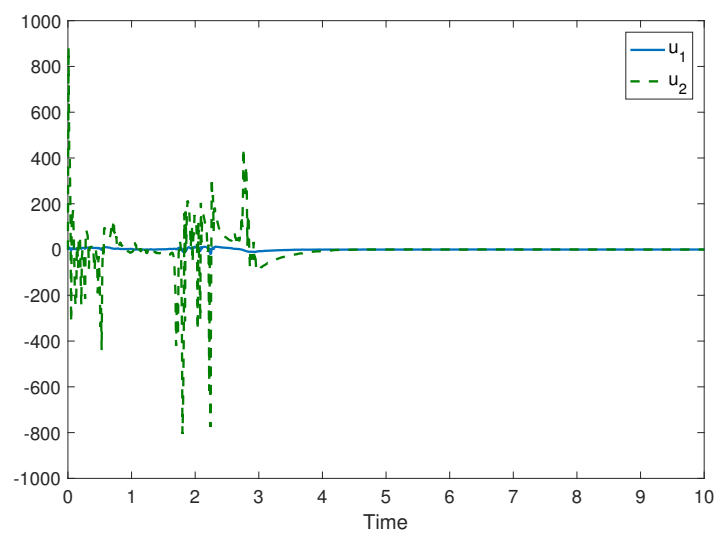

Fig. 8 The designed control inputs for the closed-loop semi-linear stochastic system with unstable $A$ matrix.

PI controller parameters have been tuned following the minimum entropy criterion, however most of the parameters we tried cannot stabilise the system outputs and the best performance obtained has been demonstrated by the following figure. Comparing with Fig. 7, the system outputs are bounded however the convergence performance cannot be guaranteed even if the parametric optimisation has been adopted.

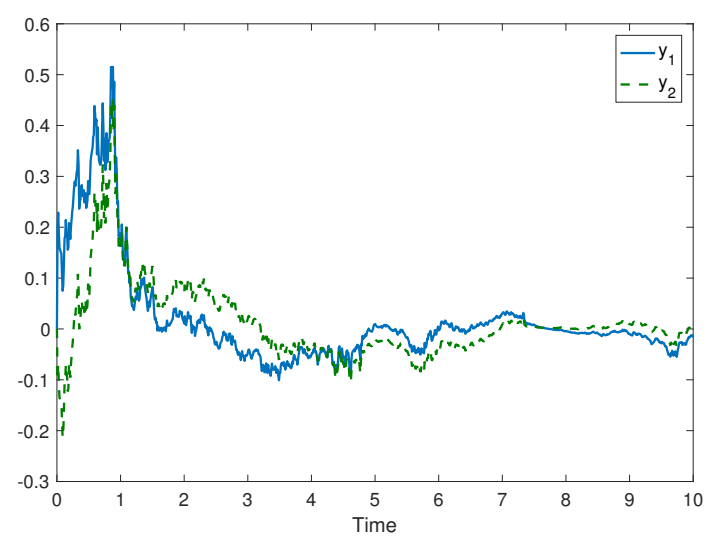

Fig. 9 The PI control inputs for the closed-loop semi-linear stochastic system with unstable $A$ matrix.

\section{Conclusions}

In this paper, the transient performance optimisation has been investigated for a class of MIMO semi-linear stochastic systems. Based on the system stabilisation and transient optimisation, a control framework has been presented, in particular, the linear estimator has been first designed which forms the entire strict-feedback format with the investigated system model. Based upon the entire closed-loop system model, the $m$-block backstepping design has been adopted with the free design parameters while the system performance can be further taken into account following the parametric optimisation of the presented controller. Basi- 
cally, the system output randomness and the probabilistic decoupling have been proposed via the entropy-based performance criterion and the mutual information based performance criterion, respectively. Following the standard gradient descent searching, the control objectives can be achieved when the performance criterion has been attenuated. Moreover, the convergence in the probabilistic sense has also been analysed following the Lyapunov method with the backstepping design. Generally, the optimisation only affects the transient performance due to the fact that the system outputs will be stabilised in the probabilistic sense based on the convergence analysis.

Notice that both presented performance criteria can be represented by the information potential which can be simply approximated using multidimensional kernel density estimation (MKDE) with sampling data. The convexity of the presented performance criteria is also analysed in the paper which guarantees that the optimum exists and the presented control framework is implementable in practice. To evaluate the presented control algorithm, a numerical example has been given and the simulation results illustrate its effectiveness and correctness. In addition, other performance criteria can further be considered following the similar approach by replacing the presented performance criteria which means that the design approach can be adopted to achieve other design requirements in the future. Motivated by finite-time convergence ${ }^{[42]}$ and $H_{2} \backslash H_{\infty}$ fault diagnosis application ${ }^{[43]}$, the presented framework extensions should also focus on theoretical performance enhancement based on the transient optimization.

\section{Acknowledgement}

We would like to thank the editor and the reviewers for their valuable comments.

\section{References}

[1] M. Krstic, I. Kanellakopoulos, and P. V. Kokotovic, Nonlinear and adaptive control design. Wiley, 1995.

[2] S.-J. Liu, J.-F. Zhang, and Z.-P. Jiang, "Decentralized adaptive output-feedback stabilization for large-scale stochastic nonlinear systems," Automatica, vol. 43, no. 2, pp. 238-251, 2007.

[3] F. Wang, Z. Liu, Y. Zhang, and C. P. Chen, "Adaptive quantized controller design via backstepping and stochastic small-gain approach," IEEE Transactions on Fuzzy Systems, vol. 24, no. 2, pp. 330-343, 2016.

[4] S. Tong, T. Wang, Y. Li, and H. Zhang, "Adaptive neural network output feedback control for stochastic nonlinear systems with unknown dead-zone and unmodeled dynamics," IEEE transactions on cybernetics, vol. 44, no. 6, pp. 910-921, 2014.

[5] X.-J. Xie and N. Duan, "Output tracking of highorder stochastic nonlinear systems with application to benchmark mechanical system," IEEE Transactions on Automatic Control, vol. 55, no. 5, pp. 1197-1202, 2010 .
[6] W. Chen, L. Jiao, J. Li, and R. Li, "Adaptive nn backstepping output-feedback control for stochastic nonlinear strict-feedback systems with time-varying delays," IEEE Transactions on Systems, Man, and Cybernetics, Part B (Cybernetics), vol. 40, no. 3, pp. 939-950, 2010.

[7] P. Jagtap and M. Zamani, "Backstepping design for incremental stability of stochastic hamiltonian systems with jumps," IEEE Transactions on Automatic Control, vol. 63, no. 1, pp. 255-261, 2018.

[8] H. Wang, K. Liu, X. Liu, B. Chen, and C. Lin, "Neural-based adaptive output-feedback control for a class of nonstrict-feedback stochastic nonlinear systems," IEEE transactions on cybernetics, vol. 45, no. 9, pp. 1977-1987, 2015.

[9] Y. Chang, "Block backstepping control of mimo systems," IEEE Transactions on Automatic Control, vol. 56, no. 5, pp. 1191-1197, 2011.

[10] C. Cheng, G. Su, and C. Chien, "Block backstepping controllers design for a class of perturbed non-linear systems with m blocks," IET Control Theory $\&$ Applications, vol. 6, no. 13, pp. 2021-2030, 2012.

[11] Q. Zhang, J. Zhou, H. Wang, and T. Chai, "Output feedback stabilization for a class of multi-variable bilinear stochastic systems with stochastic coupling attenuation," IEEE Transactions on Automatic Control, vol. 62, no. 6, pp. 2936-2942, 2017.

[12] Q. Zhang and X. Yin, "Observer-based parametric decoupling controller design for a class of multi-variable non-linear uncertain systems," Systems Science \& Control Engineering, vol. 6, no. 1, pp. 258-267, 2018.

[13] Q. Zhang, J. Zhou, H. Wang, and T. Chai, "Minimized coupling in probability sense for a class of multivariate dynamic stochastic control systems," in 201554 th IEEE Conference on Decision and Control (CDC), pp. 1846-1851, IEEE, 2015.

[14] Q. Zhang and L. Hu, "Probabilistic decoupling control for stochastic non-linear systems using ekf-based dynamic set-point adjustment," in 2018 UKACC 12th International Conference on Control (CONTROL), pp. 330-335, IEEE, 2018.

[15] Q. Zhang, Z. Wang, and H. Wang, "Parametric covariance assignment using a reduced-order closed-form covariance model," Systems Science 86 Control Engineering, vol. 4, no. 1, pp. 78-86, 2016.

[16] Q. Zhang and F. Sepulveda, "A statistical description of pairwise interaction between nerve fibres?," in 2017 8th International IEEE/EMBS Conference on Neural Engineering (NER), pp. 194-198, IEEE, 2017.

[17] Q. Zhang and F. Sepulveda, "A model study of the neural interaction via mutual coupling factor identification," in 2017 39th Annual International Conference of the IEEE Engineering in Medicine and Biology Society (EMBC), pp. 3329-3332, IEEE, 2017. 
[18] H. Wang, "Minimum entropy control of non-gaussian dynamic stochastic systems," IEEE Transactions on Automatic Control, vol. 47, no. 2, pp. 398-403, 2002.

[19] H. Yue and H. Wang, "Minimum entropy control of closed-loop tracking errors for dynamic stochastic systems," IEEE Transactions on Automatic Control, vol. 48, no. 1, pp. 118-122, 2003.

[20] M. Ren, J. Zhang, M. Jiang, M. Yu, and J. Xu, "Minimum $h-\phi$ - entropy control for non-gaussian stochastic networked control systems and its application to a networked dc motor control system," IEEE Transactions on Control Systems Technology, vol. 23, no. 1, pp. 406411, 2015.

[21] J. Zhang, L. Du, M. Ren, and G. Hou, "Minimum error entropy filter for fault detection of networked control systems," Entropy, vol. 14, no. 3, pp. 505-516, 2012.

[22] L. Guo and H. Wang, "Minimum entropy filtering for multivariate stochastic systems with non-gaussian noises," IEEE Transactions on Automatic control, vol. 51, no. 4, pp. 695-700, 2006.

[23] L. Guo, L. Yin, H. Wang, and T. Chai, "Entropy optimization filtering for fault isolation of nonlinear nongaussian stochastic systems," IEEE Transactions on Automatic Control, vol. 54, no. 4, pp. 804-810, 2009.

[24] L. Guo, H. Wang, and T.-y. Chai, "Fault detection for non-linear non-gaussian stochastic systems using entropy optimization principle," Transactions of the Institute of Measurement and Control, vol. 28, no. 2, pp. 145-161, 2006.

[25] L. Yao, J. Qin, H. Wang, and B. Jiang, "Design of new fault diagnosis and fault tolerant control scheme for non-gaussian singular stochastic distribution systems," Automatica, vol. 48, no. 9, pp. 2305-2313, 2012.

[26] M. Yang and A. Armaou, "Feedback control of semilinear distributed parameter systems using advanced pod method," in Decision and Control (CDC), 2015 IEEE 54th Annual Conference on, pp. 4680-4687, IEEE, 2015.

[27] Q. Zhang, L. Hu, and J. Gow, "Output feedback stabilization for dynamic mimo semi-linear stochastic systems with output randomness attenuation," in $A u$ tomation and Computing (ICAC), 2018 24rd International Conference on, pp. 1-6, IEEE, 2018.

[28] Q. Zhang and A. Wang, "Decoupling control in statistical sense: minimised mutual information algorithm," International Journal of Advanced Mechatronic Systems, vol. 7, no. 2, pp. 61-70, 2016.

[29] J. Bao, H. Yue, W. E. Leithead, and J.-Q. Wang, "Feedforward control for wind turbine load reduction with pseudo-lidar measurement," International Journal of Automation and Computing, vol. 15, no. 2, pp. 142-155, 2018.
[30] P. Cross and X. Ma, "Model-based and fuzzy logic approaches to condition monitoring of operational wind turbines," International Journal of Automation and Computing, vol. 12, no. 1, pp. 25-34, 2015.

[31] F. Qin, Q. Zhang, W. Zhang, Y. Yang, J. Ding, and $\mathrm{X}$. Dai, "Link quality estimation in industrial temporal fading channel with augmented kalman filter," IEEE Transactions on Industrial Informatics, 2018.

[32] C. Yang, G. Peng, Y. Li, R. Cui, L. Cheng, and Z. Li, "Neural networks enhanced adaptive admittance control of optimized robot-environment interaction," IEEE transactions on cybernetics, no. 99, pp. 1-12, 2018.

[33] C. Yang, J. Luo, Y. Pan, Z. Liu, and C.-Y. Su, "Personalized variable gain control with tremor attenuation for robot teleoperation," IEEE Transactions on Systems, Man, and Cybernetics: Systems, no. 99, pp. 1$12,2017$.

[34] R. Khasminskii, Stochastic stability of differential equations, vol. 66. Springer Science \& Business Media, 2011.

[35] T. M. Cover and J. A. Thomas, Elements of information theory. John Wiley \& Sons, 2012.

[36] Y. Zhou, Q. Zhang, H. Wang, P. Zhou, and T. Chai, "Ekf-based enhanced performance controller design for nonlinear stochastic systems," IEEE Transactions on Automatic Control, vol. 63, no. 4, pp. 1155-1162, 2018.

[37] Y. Zhou, Q. Zhang, and H. Wang, "Enhanced performance controller design for stochastic systems by adding extra state estimation onto the existing closed loop control," in Control (CONTROL), 2016 UKACC 11th International Conference on, pp. 1-6, IEEE, 2016.

[38] X. Dai, Z. Gao, T. Breikin, and H. Wang, "High-gain observer-based estimation of parameter variations with delay alignment," IEEE Transactions on Automatic Control, vol. 57, no. 3, pp. 726-732, 2012.

[39] M. Ren, Q. Zhang, and J. Zhang, "An introductory survey of probability density function control," Systems Science $\&$ Control Engineering, vol. 7, no. 1, pp. 158-170, 2019.

[40] Y. Liu, H. Wang, and C. Hou, "Ukf based nonlinear filtering using minimum entropy criterion," IEEE Transactions on Signal Processing, vol. 61, no. 20, pp. 49884999, 2013.

[41] Q. Zhang, A. Wang, and H. Wang, "Minimum mutual information control for multi-variable non-gaussian stochastic systems," in 2015 International Conference on Advanced Mechatronic Systems (ICAMechS), pp. 497-502, IEEE, 2015.

[42] C. Yang, Y. Jiang, W. He, J. Na, Z. Li, and B. Xu, "Adaptive parameter estimation and control design for robot manipulators with finite-time convergence," IEEE Transactions on Industrial Electronics, vol. 65, no. 10, pp. 8112-8123, 2018. 
[43] X. Dai, Z. Gao, T. Breikin, and H. Wang, "Zero assignment for robust $h_{2} \backslash h_{\infty}$ fault detection filter design," IEEE Transactions on Signal processing, vol. 57, no. 4, pp. 1363-1372, 2008.

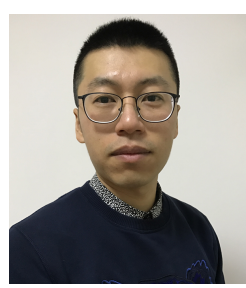

Qichun Zhang received the $\mathrm{PhD}$ degree in Electrical and Electronic Engineering from University of Manchester, UK, in 2016. He also received MSc in Control Theory and Control Engineering in 2010 and BEng in Automation in 2008, respectively, from Northeastern University, China. Currently, Dr Zhang is Senior Lecturer in Dynamics and Control at De Montfort University in Leicester, UK, where his research is supported by ViceChancellor 2020 scheme. Before joining De Montfort University in 2017, Dr Zhang was a Senior Research Officer at University of Essex, UK. From 2011-2013, He was an academic visitor at Control Systems Centre, University of Manchester. He serves over 20 international journals as an active reviewer and his current research interests include stochastic dynamic systems, probabilistic coupling analysis, decoupling control, performance optimisation, brain-computer interface and computational modelling for peripheral nervous systems.

E-mail: qichun.zhang@dmu.ac.uk (Corresponding author)

ORCID iD: 0000-0003-2479-8195

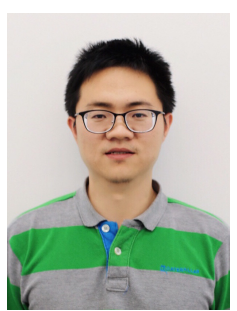

Liang $\mathbf{H u}$ received both the B.E. and M.E. degrees from Harbin Institute of Technology, China, in 2008 and 2010, respectively, and the Ph.D. degree from Brunel University London, U.K., in 2016. Dr Hu has been a lecturer in intelligent transport systems at De Montfort University, U.K since 2018. Prior to that, He did the postdoctoral research at Queen's University Belfast and Loughborough University. His research interests include signal processing, control and decision and their applications in autonomous systems and intelligent transport systems.

E-mail: liang.hu@dmu.ac.uk

ORCID iD: 0000-0002-9635-4297

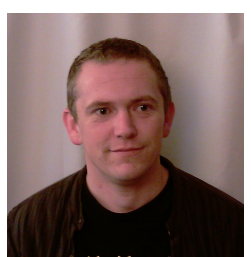

John Gow received an MEng in Electronic and Electrical Engineering from Loughborough University in 1993, and a Ph.D in power electronics (also from Loughborough University) in 1998. He subsequently continued research in the area of power conversion systems for buildingintegrated and large scale solar photovoltaic installations. Subsequent industrial opportunities in power electronics and embedded control led to him acting as a senior design engineer developing hardware and software for DSP and microcontroller based embedded control systems along with power chains for inverters, industrial drives and uninterruptible power supplies. He developed a working knowledge of RF hardware design through an industrial position developing hardware for radio frequency identification systems as well as a lifelong interest in amateur radio. He now works as Associate Professor of Electronic Engineering at De Montfort University with research interests in power electronics, high speed embedded systems for control applications and software radio.

E-mail: jgow@dmu.ac.uk

ORCID iD: 0000-0002-7288-2060 